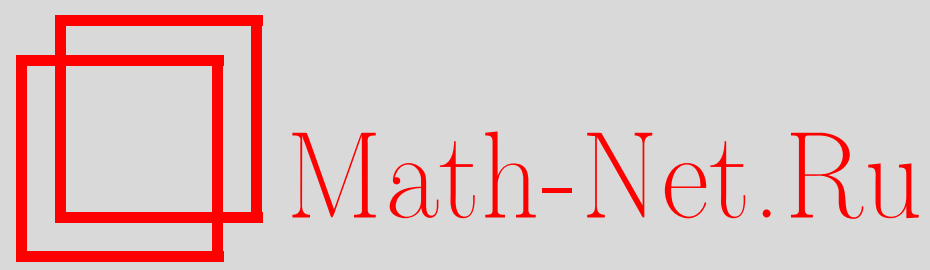

И. А. Икромов, Демпфированные осцилляторные интегралы и максимальные операторы, Матем. заметки, 2005, том 78, выпуск 6, 833-852

DOI: https://doi.org/10.4213/mzm2656

Использование Общероссийского математического портала Math-Net.Ru подразумевает, что вы прочитали и согласны с пользовательским соглашением http://www.mathnet.ru/rus/agreement

Параметры загрузки:

IP : 34.229 .108 .108

26 апреля 2023 г., 13:43:05

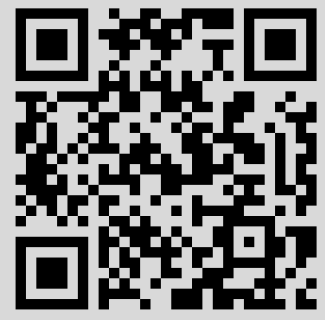




\title{
ДЕМПФИРОВАННЫЕ ОСЦИЛЛЯТОРНЫЕ ИНТЕГРАЛЫ И МАКСИМАЛЬНЫЕ ОПЕРАТОРЫ
}

\section{И. А. Икромов}

\begin{abstract}
В данной работе рассматриваются оценки преобразования Фурье борелевских мер, сосредоточенных на аналитических гиперповерхностях и содержащих множитель гашения. Множители гашения определяются через главные кривизны поверхности. Полученные оценки применяются к проблеме об ограниченности соответствующих максимальных операторов.

Библиография: 25 названий.
\end{abstract}

1. Введение. Одним из классических результатов действительного анализа является так называемая максимальная теорема Стейна о сферических средних в $\mathbb{R}^{n+1}$, $n \geqslant 2$ (см. [1]). Позднее, аналог теоремы Стейна для плоского случая был доказан Бургеном в работе [2]. Эти результаты стали отправной точкой для изучения различного класса максимальных операторов, связанных с подмногообразиями евклидова пространства. В данной работе рассматриваются максимальные операторы, определенные соотношением

$$
M g(x)=\sup _{t>0}\left|\int_{S} g(x-t y) \psi(y) d \sigma(y)\right|,
$$

где $S \subset \mathbb{R}^{n+1}$ - гладкая гиперповерхность, $\psi$ - фиксированная неотрицательная функция с компактньм носителем, $d \sigma$ - индуцированная лебегова мера на $S$ и $g \in C_{0}^{\infty}\left(\mathbb{R}^{n+1}\right)$.

Через $L^{p}:=L^{p}\left(\mathbb{R}^{n+1}\right)$ обозначается множество измеримых функций $f$, определенных в $\mathbb{R}^{n+1}$, для которых $|f(x)|^{p}$ интегрируема по $\mathbb{R}^{n+1}$.

Говорят, что оператор $M$ ограничен в $L^{p}$ (или $L^{p}$-ограничен), если существует положительное число $C$ такое, что для любого $g \in C_{0}^{\infty}\left(\mathbb{R}^{n+1}\right)$ выполняется неравенство $\|M g\|_{L^{p}} \leqslant C\|g\|_{L^{p}}$. Так как $\psi$ имеет компактньй носитель, то рассматриваемые операторы ограничены в $L^{\infty}$. Поэтому, если максимальный оператор ограничен в $L^{p}$ для некоторого конечного $p=p_{0}$, то согласно интерполяционной теореме он ограничен в $L^{p}$ для любого $p>p_{0}$ [3]. В дальнейшем через $p(S)$ обозначается минимальное число такое, что для любого $p>p(S)$ максимальный оператор (1) ограничен в $L^{p}$ (разумеется, возможен случай $p(S)=\infty$ ).

Максимальные операторы вида (1) исследовались в работах многих авторов [4]. Гринлиф [5] доказал, что если $S$ строго вьпуклая и звездная относительно начала координат гиперповерхность в $\mathbb{R}^{n+1}, n \geqslant 2$ (это означает, что любой луч исходящий из начала координат пересекается с поверхностью в единственной точке и $K(x) \neq 0$, где $K(x)$ - гауссова кривизна поверхности), и $\psi \neq 0$, то $p(S)=(n+1) / n$. Более того, как он показал, если в каждой точке поверхности $S$ имеются хотя бы $k, k \geqslant 2$, ненулевые 
главные кривизны, то $p(S) \leqslant(k+1) / k$. В более сложном случае $k=1$ аналогичньй результат получен Согги [6] .

В работе [3] Согги и Стейна доказано, что если гауссова кривизна поверхности не имеет нулей бесконечного порядка, то $p(S)<\infty$. Однако, как показьвают примеры, оценка, указанная для $p(S)$ в этой работе, далека от его точного значения. В работах [5], [7]-[10] получено точное значение $p(S)$ для некоторых выпуклых гиперповерхностей.

В общем случае проблема об ограниченности максимальных операторов до сих пор остается открытой.

Известно, что $L^{p}$-оценки максимальных операторов связаны с поведением осцилляторных интегралов следующего вида:

$$
\int_{S} e^{i(\xi, x)} \psi(x) d \sigma(x) .
$$

Точнее, чтобы получить $L^{p}$-оценки для максимального оператора, необходимо иметь равномерные (относительно направлений вектора $\xi$ ) оценки для соответствующего осцилляторного интеграла (2) при больших значениях $|\xi|$. Равномерные оценки осцилляторных интегралов исследовались в различных работах. Как известно, [11]-[13] в зависимости от особенностей фазовой функции $(\xi, x)$ убывание осцилляторного интеграла (2) на бесконечности может быть довольно медленньм. Вот почему Согги и Стейн [3] ввели следующие демпфированные осцилляторные интегралы:

$$
\int_{S} e^{i(\xi, x)}|K(x)|^{q} \psi(x) d \sigma(x)
$$

где $K(x)$ - гауссова кривизна поверхности в точке $x \in S$.

Они доказали, что если $q \geqslant 2 n$, то интеграл (3) убывает как $O\left(|\xi|^{-n / 2}\right)$ при $|\xi| \rightarrow+\infty$. Откуда с использованием свойства интегрируемости гауссовой кривизны с некоторой отрицательной степенью получена оценка для $p(S)$. Аналогичные интегралы в случае выпуклых гиперповерхностей рассмотрены в работе [14]. С другой стороны, как показьвают результаты Гринлифа и Согги [5], [6], существует гиперповерхность со свойством $p(S)<\infty$, но с нулевой гауссовой кривизной. Таким образом, условие конечности порядка всех нулей гауссовой кривизны далеко не необходимо.

\section{Отсюда возникает естественная}

ЗАдАчА. Если у гиперповерхности $S$ не все главные кривизны тождественно равны нулю, то что можно сказать об $L^{p}$-ограниченности соответствующего максимального оператора?

Ясно, что в общем случае, степень гауссовой кривизны в качестве множителя гашения не дает никаких результатов.

Паламодов обратил внимание автора к другим, более естественным семействам множителей гашения [15]. В данной работе мы рассмотрим оценку осцилляторных интегралов с множителем гашения, содержащимся в таких семействах, и получим решение проблемы ограниченности для более широкого класса максимальных операторов.

Пусть $S \subset \mathbb{R}^{n+1}$ - любая аналитическая гиперповерхность. Для точки $x \in S$ через

$$
G(x)=\left\{g_{i j}(x)\right\} \quad \text { и } \quad A(x)=\left\{a_{i j}(x)\right\}
$$


обозначаются матрицы первой и второй фундаментальной формы поверхности $S$ соответственно. Естественно определяется тензор $\left\{g^{i j}(x)\right\}[16]$.

Пусть $D$ - тензор, получаемый внешним умножением $A(x)$ на само себя: $A(x) \wedge A(x)$. Тензор $D=\left\{d_{i_{1} i_{2} i_{3} i_{4}}\right\}$ является тензором ранга $(0,4)$.

Введем функции, определенные следующими равенствами:

$$
\begin{aligned}
& \Lambda_{1}(x):=\sum g^{i_{1} j_{1}}(x) g^{i_{2} j_{2}}(x) a_{i_{1} i_{2}}(x) a_{j_{1} j_{2}}(x), \\
& \Lambda_{2}(x):=\sum g^{i_{1} j_{1}}(x) g^{i_{2} j_{2}}(x) g^{i_{3} j_{3}}(x) g^{i_{4} j_{4}}(x) d_{i_{1} i_{2} i_{3} i_{4}}(x) d_{j_{1} j_{2} j_{3} j_{4}}(x) .
\end{aligned}
$$

В этих формулах производится суммирование по всем индексам.

Заметим, что если $S$ - аналитическая гиперповерхность, то выше определенные функции аналитичны на $S$. Демпфируюшие множители (множители гашения) определяются следующей формулой:

$$
\Lambda(x):=\Lambda_{1}(x)^{q_{1} / 2} \Lambda_{2}(x)^{q_{2} / 4},
$$

где $q_{1}, q_{2}$ - фиксированные неотрицательные вещественные числа, удовлетворяющие условиям $q_{1}+q_{2}=1$ и $q_{2}>0$.

ЗАмЕчАНИЕ 1.1. В качестве $\Lambda_{1}, \Lambda_{2}$ можно взять любые ненулевые элементы идеалов $I_{1}^{2}, I_{2}^{2}$, где $I_{1}$ (соответственно $I_{2}$ ) - локальный идеал, порожденньй элементами матрицы $A(x)$ (соответственно $A(x) \wedge A(x)$ ), и по ним определить аналогичные демпфирующие множители. Однако эти множители не улучшают нашу оценку для $p(S)$.

Справедлива следующая теорема об ограниченности максимальных операторов.

Теорема 1.1. Пусть $S \subset \mathbb{R}^{n+1}$ - аналитическая гиперповерхность, $\psi \in C_{0}^{\infty}(S)-$ фиксированная неотрицательная функиия и $M$-максимальный оператор, определенный равенством (1). Если $\Lambda_{1}^{-\beta / 2} \in L_{\mathrm{loc}}^{1}(S)$ (где $\beta>0$ - фиксированное число), а также $\Lambda_{2}$ - ненулевая аналитическая функиия, то максимальный оператор $M$ ограничен в $L^{p}$ для любого $p>p(\beta):=2+3 /(2 \beta)$. Иными словами, при этих условиях справедлива оценка $p(S) \leqslant p(\beta)$.

ЗАмечАниЕ 1.2. Теорема 1.1 обобщает результат Согги и Стейна [3]. Более того, если гауссова кривизна поверхности не имеет нулей бесконечного порядка, то мы получим более точную оценку для $p(S)$. В частности, из теоремы 1.1 вытекает, что если у поверхности $S$ все главные кривизны, за исключением хотя бы двух, тождественно равны нулю, то $p(S) \leqslant p(\beta)<\infty$. Таким образом, теорема 1.1 улучшает также результат Гринлифа [5], которьй рассмотрел случай, когда в каждой точке поверхности имеются хотя бы $k, k \geqslant 2$, ненулевые главные кривизны. Отметим, что в нашем случае в некотором собственном аналитическом множестве все главные кривизны могут обратиться в нуль.

ЗАмечАниЕ 1.3 . Заметим, что если $\Lambda_{2} \equiv 0$, то согласно теореме Хартмана и Ниренберга [17] гиперповерхность имеет специальньй вид и соответствующее преобразование $\Phi$ урье с любым ненулевым множителем не может убывать быстрее $O\left(|\xi|^{-1 / 2}\right)$ при $|\xi| \rightarrow+\infty$. Следовательно, в этом случае метод демпфированных осцилляторных интегралов неприменим к проблеме об ограниченности соответствующих максимальных операторов. Хотя, если для любого $x \in S$ выполняется условие $\Lambda_{1}(x) \neq 0$, то согласно теореме Согги [6] максимальный оператор ограничен в $L^{p}$ при любом $p>2$. При этом 
если $\Lambda_{2}$ - ненулевая аналитическая функция, то в теореме 1.1 в качестве $\beta$ мы можем взять любое положительное число и получим новое доказательство теоремы Согги в этом частном случае.

ЗАмЕчАнИЕ 1.4. В общем случае трудно найти точное значение показателя $p(S)$. В работе [10] найдена связь между $p(S)$ и показателем осцилляции [11] фазовой функции в случае выпуклых гиперповерхностей. Например, если $S \subset \mathbb{R}^{4}$ задается как график функции $f(x)=x_{1}^{2 k}+x_{2}^{2 k}+x_{3}^{2 k}+1, k \geqslant 3$, и $\psi(0) \neq 0$, то из результатов Ковлинга и Маучери [8] следует, что $p(S)=2 k / 3$. В этом случае $p(\beta)=k+1$. Таким образом, мы получим значение $p(S)$ с точностью до порядка касания.

2. Сведение к демпфированным осцилляторным интегралам. Исходя из общего подхода, теорема 1.1 доказьвается методом вложения $M$ в аналитическое семейство операторов. Точнее, для $z \in \mathbb{C}, \operatorname{Re}(z)>-\beta$, определяется следующая мера (заряд):

$$
d \sigma_{z}(x)=\Lambda(x)^{z} \psi(x) d \sigma(x) \quad \text { на } S,
$$

а также соответствующий максимальный оператор:

$$
M_{z} g(x)=\sup _{t>0}\left|\int_{S} g(x-t y) d \sigma_{z}(y)\right| .
$$

Так как $\psi$ - неотрицательная функция, то для любьх $z \in \mathbb{C}$ и $g \in C_{0}^{\infty}\left(\mathbb{R}^{n+1}\right)$ вьполняется неравенство $M_{z} g(x) \leqslant M_{\operatorname{Re}(z)}|g(x)|$. Следовательно, если $\operatorname{Re}(z)=q>-\beta$ и $q_{2}$ - достаточно малое положительное число, то из условий теоремы 1.1 и неравенства Гёльдера вытекает, что $\Lambda^{q}$ - локально-интегрируемая функция. Здесь мы используем тот факт, что $\Lambda_{2}$ - ненулевая аналитическая функция и любая такая функция локально интегрируема с некоторой отрицательной степенью. Поэтому при $\operatorname{Re}(z)>-\beta$ мы имеем ограниченность оператора $M_{z}$ в пространстве $L^{\infty}$.

Если $q>3 / 2$, то ограниченность оператора $M_{q}\left(M_{z}\right)$ в пространстве $L^{2}$ вытекает из следующего утверждения и теоремы вложения Соболева [3].

ТЕОРемА 2.1. Пусть $S \subset \mathbb{R}^{n+1}$ - любая аналитическая гиперповерхность, $\psi \in$ $C_{0}^{\infty}(S)$ - любая функиия. Если $q_{1}, q_{2}, q$ - фиксированные вещественные числа maкue, чmo

$$
q_{1}+q_{2}=1, \quad q_{2}>0, \quad q>\frac{3}{2},
$$

то существует $\varepsilon>0$ такое, что для преобразования Фурье меры $d \sigma_{q}(x)$ выполняются следующие неравенства:

$$
\left|d \hat{\sigma}_{q}(\xi)\right| \leqslant \operatorname{const} \frac{\|\psi\|_{L_{3}^{1}(S)}}{|\xi|^{1 / 2+\varepsilon}}, \quad\left|\nabla d \hat{\sigma}_{q}(\xi)\right| \leqslant \operatorname{const} \frac{\|\psi\|_{L_{3}^{1}(S)}}{|\xi|^{1 / 2+\varepsilon}}
$$

где $\nabla \equiv\left(\partial / \partial \xi_{1}, \partial / \partial \xi_{2}, \ldots, \partial / \partial \xi_{n}\right),\|\cdot\|_{L_{3}^{1}(S)}$ - естественная норма пространства Соболева $L_{3}^{1}(S)$.

Как показьвают примеры, условие $q_{2}>0$ существенно. То есть, вообще говоря, при $q_{2}=0$ оценка с показателем $1 / 2+\varepsilon, \varepsilon>0$, неверна.

Наконец, доказательство теоремы 1.1 следует из $L^{\infty} \mapsto L^{\infty}$ и $L^{2} \mapsto L^{2}$ оценок с применением интерполяционной теоремы Стейна [3].

Оставшаяся часть работы посвяшена доказательству теоремы 2.1. 
3. Некоторые вспомогательные утверждения. Для оценки осцилляторных интегралов нам необходимо свойство “гладкости" амплитуды, содержащей множитель гашения. С помощью аналитичности поверхности мы установим свойство ограниченности вариации множителя гашения.

Пусть $f:\left(\mathbb{R} \times \mathbb{R}^{n}, 0\right) \mapsto(\mathbb{R}, 0)$ - вещественно-аналитическая функция. Предположим, что $f$ не равняется нулю тождественно.

Утверждение следующей леммы в случае $n=1$ легко вытекает из подготовительной теоремы Вейерштрасса-Мальгранжа [18].

ЛЕмма 3.1. Существует вещественно-аналитическое многообразие $Y$ и отображсение $\pi: Y \mapsto \mathbb{R}^{n}$, которое является композицией конечного числа б-процессов таких, что для любой точки $y^{0} \in Y$ существует карта $\left(y_{1}, \ldots, y_{n}\right)$ с иентром в точке $y^{0}$, для которой справедливо следующее соотношение:

$$
f(x, \pi(y))=y_{1}^{k_{1}} \cdots y_{n}^{k_{n}} g(x, y) p(x, y)
$$

где $g(x, y), g\left(0, y^{0}\right) \neq 0,-$ вещественно-аналитическая функция, $p(x, y)$ - унитарный псевдополином, т.е.

$$
p(x, y)=x^{m}+d_{1}(y) x^{m-1}+\cdots+d_{m}(y)
$$

здесь $d_{1}, \ldots, d_{m}$ - вещественно-аналитические функиии в точке $y^{0}$ и $d_{l}\left(y^{0}\right)=0$, $l=1, \ldots, m$.

ДокАЗАТЕЛЬСтво. Разложим функцию $f(x, s)$ в ряд Тейлора по $x$ в точке $x=0$. Имеем

$$
f(x, s)=\sum_{\nu=0}^{\infty} a_{\nu}(s) x^{k} .
$$

Существует окрестность нуля $V \subset \mathbb{C}^{n}$ такая, что для каждого $\nu$ функция $a_{\nu}(s)$ имеет аналитическое продолжение в $V$; иными словами, $a_{\nu} \in \sigma(V)$. Действительно, $f(x, s)$ как аналитическая функция в начале координат имеет аналитическое продолжение в некоторый полидиск $U \subset \mathbb{C}^{n}$ с центром в нуле, и в качестве $V$ мы можем взять ортогональную проекцию $U$ на $\mathbb{C}^{n}$.

Рассмотрим идеал $I$ кольца $\sigma(V)$, порожденный элементами $\left\{a_{\nu}\right\}_{\nu=0}^{\infty}$. Если $I=\sigma(V)$, то утверждение леммы 3.1 совпадает с утверждением обычной теоремы Вейерштрасса-Мальгранжа. Далее, предположим, что $I$ - собственный идеал. Согласно теореме Гильберта о нётеровости кольца $\sigma(V)$ идеал $I$ порождается конечным числом, скажем $N$, элементов $\left\{a_{\nu}\right\}_{\nu=0}^{N}$, где $N$ - некоторое натуральное число.

Определим функцию $c=a_{1}^{2}+\cdots+a_{N}^{2}$. Согласно теореме Хиронаки [19] о разрешении особенностей существует вещественно-аналитическое многообразие $Y$ и отображение $\pi: Y \mapsto \mathbb{R}^{n}$, которое является композицией конечного числа $\sigma$-процессов, удовлетворяющих следующему условию: для каждой точки $y^{0} \in Y$ существуют локальные координаты $\left(y_{1}, \ldots, y_{n}\right)$ с центром в этой точке, т.е. $y_{k}\left(y^{0}\right)=0, k=1, \ldots, n$, такие, что справедливо следующее соотношение:

$$
c(\pi(y))=y_{1}^{k_{1}} \cdots y_{n}^{k_{n}} b\left(y_{1}, \ldots, y_{n}\right)
$$


где $b$ - аналитическая функция в начале координат и $b(0) \neq 0$. Так как $c(\pi(y)) \geqslant 0$ для любой точки $y \in V^{\prime} \subset \mathbb{R}^{n}$, то $k_{1}, \ldots, k_{n}$ - четные неотрищательные целые числа и $b(0)>0$. Следовательно,

$$
a(y):=(c(\pi(y)))^{1 / 2}=y_{1}^{k_{1} / 2} \cdots y_{n}^{k_{n} / 2}(b(y))^{1 / 2}
$$

также является аналитической функцией в $V(0)$. Очевидно, что если $q(y)$ - аналитическая функция в $V(0)$ и $q(y) / y_{k}^{l}$ ограничена в этой же окрестности, то эта дробь имеет аналитическое продолжение в $V(0)$. Поэтому для каждого $\nu$ функция $\left(a_{\nu} / a\right)(\pi(y))$ аналитическая в некоторой фиксированной окрестности точки $y^{0}$.

Рассмотрим функцию

$$
f_{1}(x, s)=\frac{f(x, s)}{a(s)}
$$

и многообразие $Y$. Для каждой точки $y^{0} \in Y$ этого многообразия существует координатная окрестность такая, что $f_{1}(x, \pi(y))$ - вещественно-аналитическая функция в точке $\left(0, y^{0}\right)$ и при этом выполняется условие $f_{1}\left(x, \pi\left(y^{0}\right)\right) \not \equiv 0$. Следовательно, мы можем применить подготовительную теорему Вейерштрасса-Мальгранжа [18] к функции $f_{1}(x, \pi(y))$ и придем к заключению леммы 3.1 .

Лемма 3.2. Пусть $f:\left(\mathbb{R} \times \mathbb{R}^{n}, 0\right) \mapsto(\mathbb{R}, 0)$ - вещественно-аналитическая функиия. Существует окрестность нуля $U_{1} \times U_{2} \subset \mathbb{R} \times \mathbb{R}^{n}$ такая, что для любого фиксированного полоэсительного числа $q$ функиия $|f(\cdot, s)|^{q}$ имеет ограниченную вариацию по $U_{1}$ и полная вариация этой функции - $V_{U_{1}}\left[|f(\cdot, s)|^{q}\right]$ является ограниченной функиией в $U_{2}$.

ДоКАЗАТЕЛЬСТвО. Если $q \geqslant 1$, то утверждение леммы 3.2 тривиально. Поэтому нам достаточно рассмотреть случай $0<q<1$. Если $p(x)-$ полином степени $m$ и функция $F$ имеет ограниченную вариацию по отрезку $[a, b]$, то их композиция $F$ о $p(x)$ также имеет ограниченную вариацию по множеству $K:=\{x \in \mathbb{R}: a \leqslant p(x) \leqslant b\}$. Более того, справедливо неравенство

$$
V_{K}[F \circ p(x)] \leqslant m V_{a}^{b}[F] .
$$

Если функция $f$ имеет вид $f(x, s)=a(x, s) p(x, s)$, где $a(x, s)$ - аналитическая функция, удовлетворяющая условию $a(0,0) \neq 0$, и $p(x, s)$ - псевдополином, то легко видеть, что $|f(\cdot, s)|^{q}$ имеет ограниченную вариацию по $x$ как произведение функций ограниченной вариации. Если $f(x, s) \equiv 0$, то доказывать нечего. В противном случае, т.е. в случае $f(x, s) \not \equiv 0$, применяя лемму 3.1 , построим многообразие $Y$ и собственное аналитическое отображение $\pi: Y \mapsto U_{2}$ такие, что $f(x, \pi(y))$ в локальных координатах имеет вид

$$
f(x, \pi(y))=y_{1}^{k_{1}} \cdots y_{n}^{k_{n}} g(x, y) p(x, y)
$$

где $p(x, y)$ - псевдополином и $y$ - локальные координаты. Следовательно, мы получим, что $f(x, \pi(y))$ имеет ограниченную вариацию по $U_{1}$ и ее полная вариация ограничена в некоторой координатной окрестности $V$. Так как $\pi: Y \mapsto U_{2}-$ собственное аналитическое отображение, то стандартньми методами анализа мы приходим к доказательству леммы 3.2 . 
ЛЕмма 3.3. Пусть $f(x, s)$ удовлетворяет приведенным выше условиям и $0<$ $q \leqslant 1$ - фиксированное число. Тогда существует окрестность нуля $U_{1} \times U_{2} \boldsymbol{\theta}$ $\mathbb{R} \times \mathbb{R}^{n}$ такая, что функиия

$$
\varphi(x, s)=\frac{|f(x, s)|^{q}-|f(0, s)|^{q}}{|x|^{q}}
$$

имеет ограниченную вариачию по $U_{1}$ и ее полная вариачия равномерно ограничена в $U_{2}$.

ДокАЗАтЕльство. Сначала предположим, что $f(x, s)$-многочлен. Скажем, $f(x, s)$ $:=p(x, s)=x^{m}+s_{1} x^{m-1}+\cdots+s_{m}$, и коэффициенты многочлена ограничены: $|s| \leqslant 1$. Мы покажем, что функция

$$
\varphi(x, s)=\frac{|p(x, s)|^{q}-\left|s_{m}\right|^{q}}{|x|^{q}}
$$

имеет ограниченную вариацию по отрезку $[-1,1]$ и ее полная вариация $V_{-1}^{1}[\varphi(\cdot, s)]$ ограничена с константой, зависящей только от $m$. Легко доказать, что $\varphi(x, s)-$ кусочно-монотонная функция. Действительно, пусть $x>0$ и $p(x, s)>0$. Вычислим производную функции $\varphi(x, s)$ по $x$ и получим

$$
\varphi^{\prime}(x, s)=\frac{q\left(x(p(x, s))^{q-1} p^{\prime}(x, s)-\left((p(x, s))^{q}-\left|s_{m}\right|^{q}\right)\right)}{x^{q+1}} .
$$

Теперь покажем, что числитель имеет не более $2 m+1$ нулей. Выгисляя производную числителя и приравнивая ее к нулю, получим

$$
(p(x, s))^{q-2}\left((1-q) p(x, s) p^{\prime}(x, s)+x(q-1)\left(p^{\prime}(x, s)\right)^{2}+x p(x, s) p^{\prime \prime}(x, s)\right)=0 .
$$

Последнее уравнение имеет не более, чем $2 m+1$ корней.

Поэтому числитель не может иметь более, чем $2 m+2$ нулей. Отсюда следует, что уравнение $\varphi^{\prime}(x, s)=0$ имеет не более, чем $4 m+4$ корней. Заметим, что для любых чисел $x, y \in \mathbb{R}$ и $q \in(0,1]$ справедливо неравенство: $\left.|| x\right|^{q}-|y|^{q}|\leqslant| x-\left.y\right|^{q}$. Отсюда следует, что $\varphi(x, s)$ ограничена числом $m^{q}$ при $|x| \leqslant 1$.

Следовательно, имеем

$$
V_{-1}^{1}[\varphi(\cdot, s)] \leqslant(4 m+4) \max _{x \in[-1,1]}|\varphi(x, s)| \leqslant(4 m+4) m^{q} .
$$

Таким образом, полная вариация функции $\varphi$ оценивается константой, зависящей только от $m$.

Пусть теперь $f(x, s)$ - любая вещественно-аналитическая функция. В этом случае, используя лемму 3.1 , приводим нашу функцию к виду

$$
f(x, \pi(y))=y_{1}^{k_{1}} \cdots y_{n}^{k_{n}} a(x, y) p(x, y),
$$

где $a$ - аналитическая функция, удовлетворяющая условию $a(0,0) \neq 0$, и $p(x, y)$ - некоторьй псевдополином. В этом случае мы имеем соотношение

$$
\begin{aligned}
\frac{|a(x, y) p(x, y)|^{q}-|a(0, y) p(0, y)|^{q}}{|x|^{q}}= & |p(x, y)|^{q} \frac{|a(x, y)|^{q}-|a(0, y)|^{q}}{|x|^{q}} \\
& +|a(0, y)|^{q} \frac{|p(x, y)|^{q}-|p(0, y)|^{q}}{|x|^{q}}
\end{aligned}
$$


Заметим, что функция $\left(|a(x, y)|^{q}-|a(0, y)|^{q}\right) /|x|^{q}$ имеет ограниченную вариацию, так как $a(0,0) \neq 0$. А также согласно лемме $3.2|p(x, y)|^{q}$ имеет ограниченную вариацию в координатной окрестности $V$. Наконец, заметим, что $\pi: Y \mapsto U_{2}$ - собственное аналитическое отображение. Отсюда стандартными методами анализа мы приходим к доказательству леммы 3.3 .

4. О разбиении поверхности. Пусть $S(\eta) \subset \mathbb{R}^{n+1}$ - семейство гладких гиперповерхностей, где $\eta \in V \subset \mathbb{R}^{m}$ и $V$ - некоторая достаточно малая окрестность начала координат в $\mathbb{R}^{m}$. Мы имеем дело с локальной задачей. Поэтому мы можем фиксировать систему координат в $\mathbb{R}^{n+1}$. Более того, для каждого фиксированного значения параметра $\eta \in V$ имеется взаимно-однозначное соответствие между точками $U \subset \mathbb{R}^{n}$ и точками гиперповерхности $S(\eta)$.

Наш метод оценки осцилляторных интегралов основан на разбиении гиперповерхности $S$ или семейства гиперповерхностей $S(\eta)$ с использованием главных кривизн поверхности $S(S(\eta))$. Аналогичное разбиение с использованием гауссовой кривизны рассмотрено в работе [3].

Пусть $X_{0} \in S(0)$ - фиксированная точка поверхности. Мы рассмотрим покрытые множества

$$
S(\eta) \cap\left\{X \in S(\eta): \Lambda_{1}(X, \eta) \neq 0\right\}
$$

в достаточно малой окрестности точки $X_{0}$, где

$$
\Lambda_{1}(x) \equiv \sum g^{i_{1} j_{1}}(x) g^{i_{2} j_{2}}(x) a_{i_{1} i_{2}}(x) a_{j_{1} j_{2}}(x) .
$$

Без ограничения общности мы можем предполагать, что семейство гиперповерхностей $S(\eta)$ задано как график семейства гладких функций $f(\cdot, \eta)$ и $X(0)=(0,0)$, $d_{x} f(0,0)=0$. Таким образом, мы имеем

$$
S(\eta)=\left\{X=(x, f(x, \eta)) \in \mathbb{R}^{n+1}: x \in U\right\},
$$

где $U$ - окрестность нуля в $\mathbb{R}^{n}$. На самом деле, мы рассмотрим покрытые множества

$$
U_{0}(\eta)=U \cap \pi_{n}\left(\left\{X \in S(\eta): \Lambda_{1}(X, \eta) \neq 0\right\}\right),
$$

где $\pi_{n}: \mathbb{R}^{n+1} \mapsto \mathbb{R}^{n}$ - проекция вдоль оси $x_{n+1}$.

Обозначим через $\lambda_{1}(x, \eta), \ldots, \lambda_{n}(x, \eta)$ главные кривизны поверхности $S(\eta)$ в точке $X(\eta):=(x, f(x, \eta))$.

Далее, через $c, c_{1}, \ldots, c_{5}$ обозначаются постоянные, не зависящие от параметров $\delta, \eta$, $0<\delta \leqslant 1$ (где $\delta \asymp|\lambda|$ - радиус шара), и $t, 0<t<\infty(t-$ параметр осцилляции).

Постоянные, обозначаемые одними и теми же буквами, могут быть различными в разных местах.

Не умаляя общности, мы можем считать, что

$$
\left|\lambda_{1}(x, \eta)\right|=\max _{1 \leqslant l \leqslant n}\left\{\left|\lambda_{l}(x, \eta)\right|\right\} .
$$

Через $B_{x}:=B\left(x, c\left|\lambda_{1}(x, \eta)\right|\right)$ обозначим открытый шар радиуса $c\left|\lambda_{1}(x, \eta)\right|$ с центром в точке $x$, где $c$ - достаточно малое положительное число. Для функций из класса $C^{\infty}(\bar{U} \times \bar{V})$ введем норму $\|\cdot\|_{n}$ следующим соотношением:

$$
\|f\|_{n}=\max _{\eta \in \bar{V}}\|f(\cdot, \eta)\|_{C^{n}(\bar{U})},
$$

где $\|\cdot\|_{C^{n}(\bar{U})}-$ естественная норма пространства $\mathbb{C}^{n}(\bar{U})$.

В дальнейшем ради упрошения обозначений мы будем считать, что функции дифференцируемы в некотором компактном множестве, содержащем множество $U \times V$. 
Лемма 4.1. Существуют окрестность $U \times V \subset \mathbb{R}^{n} \times \mathbb{R}^{m}$, полохсительные постоянные $c, c_{1}, c_{2}$, зависящие только от $\|f\|_{5}$, такие, что для любы $(x, \eta) \in U \times V$ и $y \in B_{x}$ выполняются следуюшие неравенства:

$$
c_{1}\left|\lambda_{1}(x, \eta)\right| \leqslant\left|\lambda_{1}(y, \eta)\right| \leqslant c_{2}\left|\lambda_{1}(x, \eta)\right|
$$

ДокАЗАТЕЛЬСТво. Если $\lambda_{1}(x, \eta)=0$ для некоторой точки $(x, \eta) \in U \times V$, то доказьвать нечего, так как в этом случае $B_{x}=\varnothing$.

В противном случае вблизи точки $x$ представим функцию $f(y, \eta)$ по формуле Тейлоpa:

$f(y, \eta)=f(x, \eta)+\left(\nabla_{x} f(x, \eta), x-y\right)+\left(D_{x}^{2} f(x, \eta)(x-y), x-y\right)+\sum_{|\alpha|=3}(x-y)^{\alpha} H_{\alpha}(x, y, \eta)$,

где $\alpha \in \mathbb{Z}_{+}^{n}$ - мультииндекс, $|\alpha|=\alpha_{1}+\cdots+\alpha_{n}, H_{\alpha} \in C^{\infty}$. Заметим, что $\|\cdot\|_{k \text {-нормы }}$ функции $H_{\alpha}$ оцениваются через $\|\cdot\|_{k+3}$-нормы функции $f$.

Легко видеть, что если $|x-y|<c\left|\lambda_{1}(x, \eta)\right|$, то для любой точки $y \in B_{x}$ вьполняются следующие неравенства:

$$
\left|\partial_{i j}^{2} \sum_{|\alpha|=3}(x-y)^{\alpha} H_{\alpha}(x, y, \eta)\right| \leqslant C c\left|\lambda_{1}(x, \eta)\right|, \quad i, j=1, \ldots, n,
$$

где $\partial_{i j}^{2}$ - повторное дифференцирование по $x_{i}$ и $x_{j}, C$ - постоянное число, зависящее только от $\|f\|_{5}$. Таким образом, для любой точки $y \in B_{x}$ вьполняется неравенство

$$
\left\|D_{y}^{2} f(y, \eta)\right\| \leqslant C\left|\lambda_{1}(x, \eta)\right|
$$

здесь $\left\|D_{y}^{2} f(y, \eta)\right\|=\left(\Lambda_{1}(y, \eta)^{1 / 2}\right.$. Заметим, что для любой точки $y \in U$ имеет место неравенство

$$
\left|\lambda_{1}(y, \eta)\right| \leqslant\left\|D_{y}^{2} f(y, \eta)\right\| .
$$

Эти неравенства позволяют получить верхнюю оценку для $\left|\lambda_{1}(y, \eta)\right|$ :

$$
\left|\lambda_{1}(y, \eta)\right| \leqslant c_{2}\left|\lambda_{1}(x, \eta)\right|
$$

где $c_{2}$ - положительное число, зависящее только от $\|f\|_{5}$. Мы можем выбрать $c_{2}=C$, где $C$ - некоторое число, удовлетворяющее оценке (4.3).

Далее, мы рассмотрим оценку снизу для $\left|\lambda_{1}(y, \eta)\right|$. Пусть $G(x, \eta)$ - матрица первой фундаментальной формы (матрица метрического тензора). В нашем случае мы имеем

$$
G_{i j}(x, \eta)=\delta_{i j}+f_{x_{i}}(x, \eta) f_{x_{j}}(x, \eta), \quad i, j=1, \ldots, n,
$$

где $\delta_{i j}$ - символ Кронекера.

Следует отметить, что для любых точек $(x, \eta) \in U \times V$ и $\xi \in \mathbb{R}^{n}$ вьполняются следующие неравенства:

$$
|\xi|^{2} \leqslant(G(x, \eta) \xi, \xi) \leqslant c_{3}|\xi|^{2}
$$

где $c_{3}$ - некоторое число, зависящее только от $\nabla f(x, \eta)$, и $|\xi|^{2}=\xi_{1}^{2}+\cdots+\xi_{n}^{2}$. 
С другой стороны, для любой фиксированной точки $(x, \eta) \in U \times V$ существует вектор $\xi_{x, \eta}$, удовлетворяющий условиям $\left(G(x, \eta) \xi_{x, \eta}, \xi_{x, \eta}\right)=1$ и

$$
\left|\lambda_{1}(x, \eta)\right|=\left|\frac{\left(D_{x}^{2} f(x, \eta) \xi_{x, \eta}, \xi_{x, \eta}\right)}{\left(1+\left|\nabla_{x} f(x, \eta)\right|^{2}\right)^{1 / 2}}\right| .
$$

Согласно неравенству (4.4) для любых точек $(y, \eta) \in U \times V,(x, \eta) \in U \times V$ и $\xi \in \mathbb{R}^{n}$ мы имеем

$$
c_{3}^{-1}(G(y, \eta) \xi, \xi) \leqslant(G(x, \eta) \xi, \xi) \leqslant c_{3}(G(y, \eta) \xi, \xi) .
$$

Отсюда, используя принцип мини-макса и теорему Лагранжа о конечном приращении, получим

$$
\begin{aligned}
\left|\lambda_{1}(y, \eta)\right| \geqslant & c_{3}^{-1}\left|\frac{\left(D_{y}^{2} f(y, \eta) \xi_{x, \eta}, \xi_{x, \eta}\right)}{\left(1+\left|\nabla_{y} f(y, \eta)\right|^{2}\right)^{1 / 2}}\right| \\
\geqslant & c_{3}^{-1}\left|\frac{\left(D_{x}^{2} f(x, \eta) \xi_{x, \eta}, \xi_{x, \eta}\right)}{\left(1+\left|\nabla_{x} f(x, \eta)\right|^{2}\right)^{1 / 2}}\right| \\
& \quad-c_{3}^{-1}\left|\left(\left(\frac{D_{y}^{2} f(y, \eta) \xi_{x, \eta}}{\left(1+\left|\nabla_{y} f(y, \eta)\right|^{2}\right)^{1 / 2}}-\frac{D_{x}^{2} f(x, \eta) \xi_{x, \eta}}{\left(1+\left|\nabla_{x} f(x, \eta)\right|^{2}\right)^{1 / 2}}\right), \xi_{x, \eta}\right)\right| \\
\geqslant & c_{3}^{-1}\left(1-c_{4} c\right)\left|\lambda_{1}(x, \eta)\right| .
\end{aligned}
$$

В последнем неравенстве постоянная $c_{4}$ зависит только от $\|f\|_{3}$. Теперь выбирая $c$ так, чтобы выполнялось неравенство $c_{4} c<1$, мы придем к доказательству леммы 4.1.

Введем обозначения

$$
U_{0} \times V_{0}=\left\{(x, \eta) \in U \times V: \Lambda_{1}(x, \eta) \neq 0\right\}, \quad U_{0}(\eta)=\left\{x \in U: \Lambda_{1}(x, \eta) \neq 0\right\} .
$$

ЛЕмма 4.2. Существуют положительное число с, не зависящее от параметра $\eta \in V$, и семейство шаров

$$
\left\{B_{k}\right\}=\left\{B\left(x^{k}, c\left|\lambda_{1}\left(x^{k}, \eta\right)\right|\right)\right\}
$$

обладающее следующими свойствами:

1) $U_{0}(\eta) \subset \bigcup_{k} B_{k}^{\prime}$, где $B_{k}^{\prime}=B\left(x^{k}, 3 c\left|\lambda_{1}\left(x^{k}, \eta\right)\right|\right)$;

2) существует натуральное число $N$, не зависящее от $\eta \in V$, такое, что ни одна точка множества $U_{0}(\eta)$ не содержится в более чем $N$ иарах $B_{k}^{\prime}$;

3) $\sum_{k}\left|\lambda_{1}\left(x^{k}, \eta\right)\right|^{n}$ ряд сходится, и его сумма оценивается постоянной, не зависящей от $\eta$.

ДоКАЗАТЕЛЬСТвО. Предположим, что число $4 c$ удовлетворяет утверждению леммы 4.1. Напомним, что согласно этой лемме оно не зависит от параметра $\eta$.

Если $U_{0}(\eta)=\varnothing$, то доказывать нечего. Предположим, что $U_{0}(\eta) \neq \varnothing$. Далее, ради простоты обозначения вместо $U_{0}(\eta)$ и $\lambda_{1}(x, \eta)$ мы пишем соответственно $U_{0}$ и $\lambda_{1}(x)$. Заметим, что $\lambda_{1}$ - непрерьвная функция. Мы выберем семейство шаров с помощью следующего процесса. Через $B_{1}$ обозначим шар $B\left(x^{1}, c\left|\lambda_{1}\left(x^{1}\right)\right|\right)$, где точка $x^{1}$ выбрана из условия

$$
\left|\lambda_{1}\left(x^{1}\right)\right| \geqslant \frac{1}{2} \sup _{x \in U_{0}}\left|\lambda_{1}(x)\right| .
$$


Очевидно, точка $x^{1}$ с таким свойством существует.

Пусть $B_{1}, B_{2}, \ldots, B_{k}, k \geqslant 1$, выбраны. Мы будем выбирать $B_{k+1}$ следующим образом: если $U_{0} \backslash \bigcup_{l=1}^{k} B_{l}=\varnothing$ или $U_{0} \backslash \bigcup_{l=1}^{k} B_{l} \neq \varnothing$ и для любой точки $x \in U_{0} \backslash \bigcup_{l=1}^{k} B_{l}$ выполняется соотношение

$$
\bigcup_{l=1}^{k} B_{l} \cap B\left(x, c\left|\lambda_{1}(x)\right|\right) \neq \varnothing,
$$

то мы закончим наш процесс; при этом $\left\{B_{l}\right\}_{l=1}^{k}-$ искомое семейство шаров. В противном случае мы возьмем шар

$$
B_{k+1}=B\left(x^{k+1}, c\left|\lambda_{1}\left(x^{k+1}\right)\right|\right),
$$

где точка $x^{k+1}$ найдется из условия

$$
\left|\lambda_{1}\left(x^{k+1}\right)\right| \geqslant \frac{1}{2} \sup \left|\lambda_{1}(x)\right|
$$

здесь супремум берется по множеству

$$
\left\{x \in U_{0}: \bigcup_{l=1}^{k} B_{l} \cap B\left(x, c\left|\lambda_{1}(x)\right|\right) \neq \varnothing\right\} .
$$

Таким образом, мы определим шар $B_{k+1}=B\left(x^{k}, c\left|\lambda_{1}\left(x^{k+1}\right)\right|\right)$, выбирая точку $x^{k+1}$ из условия $\left(4.5^{\prime}\right)$.

Заметим, что последовательность шаров $\left\{B_{k}\right\}$ может быть конечной или бесконечной. Если семейство содержит бесконечное число шаров, то $\lambda_{1}\left(x^{k}\right) \rightarrow 0$ при $k \rightarrow \infty$, так как $U_{0}(\eta) \subset U$ и $U$ - ограниченное множество. Пусть $\left\{B_{k}\right\}$ - выбранное семейство шаров и $\left\{x^{k}\right\}$ соответствующая последовательность точек. Определим новое семейство шаров $\left\{B_{k}^{\prime}\right\}$ следующим образом: шар $B_{k}^{\prime}$ концентричен с шаром $B_{k}$ и имеет радиус $3 c\left|\lambda_{1}\left(x^{k}\right)\right|$. Покажем справедливость включения $U_{0} \subset \bigcup_{k} B_{k}^{\prime}$. Пусть $x \in U_{0}$ - фиксированная точка. Если $x \in B_{k}$ для некоторого $k$, то доказьвать нечего. Предположим, что $x \in U_{0} \backslash \bigcup_{k} B_{k}$. Рассмотрим шар $B_{x}$ с центром в точке $x$, имеющий радиус $c\left|\lambda_{1}(x)\right|$. Покажем, что существует шар $B_{k}$ такой, что $B_{x} \cap B_{k} \neq \varnothing$. Если семейство шаров содержит конечное число шаров, то существование такого шара следует из определения этого семейства. В противном случае, если такой шар не существует, то для любого номера $k$ имеет место неравенство

$$
\left|\lambda_{1}\left(x^{k}\right)\right| \geqslant \frac{1}{2}\left|\lambda_{1}(x)\right|>0 .
$$

Но если семейство содержит бесконечное число шаров, то $\lambda_{1}\left(x^{k}\right) \rightarrow 0$ при $k \rightarrow \infty$. Поэтому неравенство (4.6) не может вьполняться для всех номеров $k$. Это противоречие доказьвает существование шара $B_{k}$ с вьшеуказанным свойством. Пусть $k$ - минимальное число с таким свойством. Таким образом, мы имеем номер $k$ такой, что

$$
\bigcup_{l=1}^{k-1} B_{l} \cap B_{x}=\varnothing, \quad B_{x} \cap B_{k} \neq \varnothing .
$$

Легко показать справедливость неравенства $\left|\lambda_{1}(x)\right| \leqslant 2\left|\lambda_{1}\left(x^{k}\right)\right|$. Следовательно,

$$
B\left(x^{k}, c\left|\lambda_{1}\left(x^{k}\right)\right|\right) \cap B\left(x, 2 c\left|\lambda_{1}\left(x^{k}\right)\right|\right) \neq \varnothing .
$$


В результате получим $x \in B_{k}^{\prime}:=B\left(x, 3 c\left|\lambda_{1}\left(x^{k}\right)\right|\right)$.

Наконец, покажем справедливость свойства конечности покрытия. Существует натуральное число $N$, не зависящее от $\eta \in V$, такое, что ни одна точка множества $U_{0}(\eta)$ не содержится в более, чем $N$ шаров $B_{k}^{\prime}$. Следуя [3], предположим, что $B_{1}^{\prime}, B_{2}^{\prime}, \ldots, B_{N}^{\prime}$ содержат $\bar{x}$. Тогда согласно лемме 4.1 их радиусы сравнимы с $\left|\lambda_{1}(\bar{x})\right|$; при этом объединение этих шаров содержится в шаре $\widetilde{B}$ с центром в точке $\bar{x}$, имеющем радиус, сравнимый с $\left|\lambda_{1}(\bar{x})\right|$. Заметим, что шары $B_{1}, B_{2}, \ldots, B_{N}$ взаимно не пересекаются, их радиусы также сравнимы с $\left|\lambda_{1}(\bar{x})\right|$ и все они содержатся в $\widetilde{B}$. Сравнивая объемы $\bigcup_{k=1}^{N} B_{k}$ и $\widetilde{B}$, получим верхнюю оценку для числа $N$. Фактически используя лемму 4.1 , элементарными рассуждениями можно показать справедливость неравенства $N \leqslant 6^{n}\left(c_{2} / c_{1}\right)^{n}$. Более того, постоянные $c_{1}, c_{2}$, определенные согласно лемме 4.1 , не зависят от $\eta$. Таким образом, утверждение 2) доказано.

Утверждение 3) леммы 4.2 легко доказывается. На самом деле, сумма $\sum_{k}\left|\lambda_{1}\left(x^{k}\right)\right|^{n}$ оценивается через $\sigma(U) / \omega_{n}+$ const, где $\sigma(U)$ - мера множества $U$ и $\omega_{n}-$ объем единичного шара в $\mathbb{R}^{n}$.

Теперь рассмотрим некоторый вариант леммы Морса с параметрами [11]. Пусть $f(x, \lambda, \eta)$ - семейство гладких функций в некоторой окрестности начала координат, имеющее вид

$$
f(x, \lambda, \eta)=x_{1}^{2}+\sum_{j=2}^{n} \lambda_{j} x_{j}^{2}+\sum_{|\alpha|=3} x^{\alpha} H_{\alpha}(x, \lambda, \eta),
$$

где $\left|\lambda_{j}\right| \leqslant 1, j=2, \ldots, n, \alpha \in \mathbb{Z}_{+}^{n},|\alpha|=\alpha_{1}+\cdots+\alpha_{n},\left\{H_{\alpha}\right\}$ - некоторое семейство гладких функций.

Рассмотрим новое семейство функций, определенное равенством

$$
\Phi(x, \lambda, \eta, s)=f(x, \lambda, \eta)+s x .
$$

Теперь сформулируем и докажем некоторое вспомогательное утверждение.

Лемма 4.3. Существуют положительные числа $C, c, \varepsilon$, зависящие только от $\left\|H_{\alpha}\right\|_{2} u \lambda$, такие, что справедливы следующие утверждения:

1) если $\left|s_{1}\right|<\varepsilon$, то существует отображсение

$$
H:\left\{\left|x_{1}\right|<C c\right\} \times\left\{\left|x^{\prime}\right|<c\right\} \mapsto U_{1} \times\left\{\left|x^{\prime}\right|<c\right\}
$$

вида $H=\left(H_{1}, \ldots, H_{n}\right), H_{1}=H_{1}\left(x, s_{1}\right), H_{l}=x_{l}, l=2, \ldots, n$, удовлетворяющее условиям $H(x, 0) \equiv x и$

$$
H^{*} \Phi(x, \eta, \lambda, s)=x_{1}^{2}+\Phi_{1}\left(x^{\prime}, \eta, \lambda, s_{1}\right)+\left(s^{\prime}, x^{\prime}\right),
$$

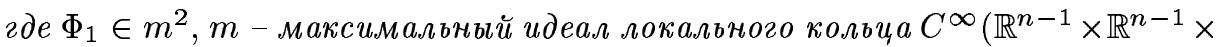
$\left.\mathbb{R}^{m} \times \mathbb{R}\right)$ в нуле и $U_{1}$ - окрестность начала координат в $\mathbb{R} ;$

2) если $\left|s_{1}\right| \geqslant \varepsilon$ и $x \in B(0, c)$, то справедливо неравенство

$$
\left|\frac{\partial \Phi}{\partial x_{1}}\right| \geqslant \frac{1}{2}\left|s_{1}\right| \text {. }
$$


ДокАЗАТЕЛЬСтво. 1) Следуя [20], рассмотрим уравнение

$$
\frac{\partial \Phi}{\partial x_{1}}=2 x_{1}+\sum_{|\alpha|=2} x^{\alpha} \widetilde{H}_{\alpha}(x, \lambda, \eta)+s_{1}=0 .
$$

Легко видеть, что существуют положительные числа $C_{1}, C_{1}^{\prime}, \varepsilon$ такие, что при $\left|s_{1}\right|<\varepsilon$ и $\left|x^{\prime}\right|<C_{1}^{\prime}$ последнее уравнение имеет решение $x_{1}(X, \lambda, \eta) \in\left(-C_{1}, C_{1}\right)$ вида

$$
x_{1}(X, \lambda, \eta)=-\frac{s_{1}}{2}+\sum_{|\alpha|=2} X^{\alpha} H_{\alpha}(X, \lambda, \eta),
$$

где $X=\left(s_{1}, x_{2}, \ldots, x_{n}\right)$.

Заметим, что $C^{k}$-нормы функции $H_{\alpha}$ оценивается через $C^{k+2}$-нормы функции $\Phi$. Теперь используем замену переменных

$$
y_{1}=x_{1}-x_{1}(X, \lambda, \eta), \quad y^{\prime}=x^{\prime} .
$$

Тогда фазовая функция $\Phi\left(x, \lambda, \eta, s_{1}\right)$ приводится к виду

$$
\Phi_{0}\left(y, \lambda, \eta, s_{1}\right)=y_{1}^{2} \Phi_{2}\left(y, \lambda, \eta, s_{1}\right)+\sum_{j=2}^{n} \lambda_{j} y_{j}^{2}+\Phi_{1}\left(y^{\prime}, \lambda, \eta, s_{1}\right)+\left(s^{\prime}, y^{\prime}\right),
$$

где $\Phi_{1}, \Phi_{2}$ - гладкие функции, удовлетворяющие условиям $\Phi_{2}(0,0,0,0)=1, \Phi_{1} \in m^{2}$ и $s^{\prime}=\left(s_{2}, \ldots, s_{n}\right), y^{\prime}=\left(y_{2}, \ldots, y_{n}\right)$.

Постоянные $C_{1}, C_{1}^{\prime}, \varepsilon$ могут быть выбраны одинаковыми в множестве функций $\left\{H_{\alpha}\right\}_{|\alpha|=3}$ и $\lambda$, удовлетворяюших условиям

$$
\left\|H_{\alpha}\right\|_{2} \leqslant M, \quad|\alpha|=3, \quad|\lambda| \leqslant 1 .
$$

Наконец, применяя замену переменных

$$
z_{1}=y_{1}\left(\Phi_{2}\left(y, \lambda, \eta, s_{1}\right)\right)^{1 / 2}, \quad z^{\prime}=y^{\prime}
$$

и обозначая через $H$ композицию с этой заменой, получим

$$
H^{*} \Phi(z, \eta, \lambda, s)=z_{1}^{2}+\Phi_{1}\left(z^{\prime}, \eta, \lambda, s_{1}\right)+\left(s^{\prime}, z^{\prime}\right) .
$$

2) Пусть теперь $\left|s_{1}\right| \geqslant \varepsilon$. Очевидно, существует положительное число $C_{2}$, зависящее от $C^{3}$-нормы функции $f$, такое, что справедливо неравенство

$$
\left|\frac{\partial f}{\partial x_{1}}\right| \leqslant C_{2} c \quad \text { при всех } x \in B(0, c) .
$$

Следовательно, если $c=\varepsilon /\left(2 C_{2}\right)$, то выполняется неравенство $\left|\partial \Phi / \partial x_{1}\right| \geqslant\left|s_{1}\right| / 2$ для любой точки $x \in B(0, c)$.

Наконец, выбирая $c=\min \left\{\varepsilon /\left(2 C_{2}\right), 2 C_{1}, C_{1}^{\prime}\right\}$ и $C=2 C_{1} / c>0$, придем к доказательству леммы 4.3.

Пусть $f(x, \eta)$ - вещественно-аналитическая функция в начале координат $\mathbb{R}^{n} \times \mathbb{R}^{m}$. Введем идеал $I$ кольца ростков аналитических функций в начало координат, порожденный элементами матрицы $D_{x}^{2} f(x, \eta)$. Корень этого идеала обозначается через $K(I)$.

В дальнейшем нам понадобится следуюшая лемма. 
ЛЕмма 4.4. Пусть $\varphi \in I$ - фиксированный әлемент идеала и $q>0$ - фиксированное полохительное число. Существуют окрестность нуля $U \times V \subset \mathbb{R}^{n} \times \mathbb{R}^{m} u$ полохсительное число с такие, что для любой точки $(x, \eta) \in U \times V \backslash K(I)$ функция

$$
\left|\frac{\varphi\left(x+\left\|D_{x}^{2} f(x, \eta)\right\| y, \eta\right)}{\left\|D_{x}^{2} f(x, \eta)\right\|}\right|^{q}
$$

имеет ограниченную вариацию по переменной $y_{l} \in[-c, c]$ и ее полная вариация по множеству $\left\{y_{l}:\left|y_{l}\right| \leqslant c\right\}$ равномерно ограничена относительно $y^{\prime} \in\left\{y^{\prime} \in \mathbb{R}^{n-1}\right.$ : $\left.\left|y_{k}\right| \leqslant c, k \neq l\right\},(x, \eta) \in U \times V \backslash K(I)$.

ДокАЗАТЕЛьСтво. Допустим, что $I \neq 0$; иначе утверждение леммы 4.4 тривиально. Далее, ради упрощения рассуждений предположим, что функция $\varphi(x, \eta)$ имеет вид

$$
\varphi(x, \eta)=g(x, \eta) \partial_{i j}^{2} f(x, \eta)
$$

В общем случае рассуждения те же самые, но мы имеем дело с более громоздкими формулами.

Рассмотрим функцию $\varphi(z, \eta)$ в множестве $\left\{z:|x-z|<c\left\|D_{x}^{2} f(x, \eta)\right\|\right\}$, где $(x, \eta) \in$ $U \times V \backslash K(I)$ и $c$ - достаточно малое положительное число, оно будет выбрано позже.

Теперь мы определим новую функцию от переменных $(y, \eta, x)$ по следующей формуле:

$$
\psi(y, \eta, x)=g\left(x+\left\|D_{x}^{2} f(x, \eta)\right\| y, \eta\right) \frac{\partial_{i j}^{2} f\left(x+\left\|D_{x}^{2} f(x, \eta)\right\| y, \eta\right)}{\left\|D_{x}^{2} f(x, \eta)\right\|},
$$

где $|y| \leqslant c$. Тогда функция

$$
\frac{\partial_{i j}^{2} f\left(x+\left\|D_{x}^{2} f(x, \eta)\right\| y, \eta\right)}{\left\|D_{x}^{2} f(x, \eta)\right\|}
$$

может быть представлена следующим соотношением:

$$
\frac{\partial_{i j}^{2} f(x, \eta)}{\left\|D_{x}^{2} f(x, \eta)\right\|}+\sum_{k=1}^{n} y_{k} a_{k}\left(\left\|D_{x}^{2} f(x, \eta)\right\| y, x, \eta\right)
$$

здесь $a_{k}, k=1, \ldots, n,-$ вещественно-аналитические функции.

Теперь, введем новые переменные

$$
\beta=\left\|D_{x}^{2} f(x, \eta)\right\|, \quad \gamma=\frac{\partial_{i j}^{2} f(x, \eta)}{\left\|D_{x}^{2} f(x, \eta)\right\|} .
$$

Легко видеть, что $\psi$ может быть рассмотрена как аналитическая функция от переменных $(x, y, \eta, \beta, \gamma)$. Более того, новые переменные $\beta, \gamma$ принадлежат некоторому компактному множеству.

Наконец, фиксируя $l \in\{1, \ldots, n\}$ и применяя лемму 3.3 к новой функции $\mid \psi(x, y, \eta$, $\beta, \gamma)\left.\right|^{q}$ относительно $y_{l}$ в множестве $\left\{y_{l}:\left|y_{l}\right| \leqslant c\right\}$ (число $c$ выбирается согласно лемме 3.3), мы придем к заключению леммы 4.4 .

ЗАмЕчАнИЕ 4.1. Отметим, что существует положительное число $c$, удовлетворяющее утверждениям лемм 4.1-4.4. 
5. Об оценках демпфированных осцилляторных интегралов. В этом пункте мы докажем теорему 2.1. Через $U_{0}$ обозначим множество $\left\{X \in S: \Lambda_{2}(X) \neq 0\right\}$. Далее, предположим, что постоянная $c$ удовлетворяет условиям лемм 4.2-4.4. Пусть $\left\{B_{k}\right\}$ - семейство шаров, построенное согласно лемме 4.2. Причем $U_{0} \subset \cup B_{k}^{\prime}$, где $B_{k}^{\prime}-$ конщентричный с $B_{k}$ шар, имеющий радиус $3 r\left(B_{k}\right)$. Кроме того, мы введем еще одно семейство концентричных с $B_{k}$ шаров $B_{k}^{\prime \prime}$ с радиусом $4 r\left(B_{k}\right)$. Рассмотрим разбиение единищы $\left\{\psi_{k}\right\}$, соответствующее покрытию $\left\{B_{k}^{\prime \prime}\right\}$. Таким образом, $\psi_{k} \in C_{0}^{\infty}\left(B_{k}^{\prime \prime}\right)$. C помощью этого разбиения единицы осцилляторньй интеграл $I(t, s)$ представляется в виде суммы интегралов

$$
I_{k}(t, s)=\int_{\mathbb{R}^{n}} e^{i t F(x, s)}(\Lambda(x))^{q} \psi(x) \psi_{k}(x) d x .
$$

Теперь рассмотрим оценку одного из этих интегралов $I_{k}(t, s)$. Следуя [3], для каждого шара $B:=B_{k}^{\prime}$ выберем систему координат в $\mathbb{R}^{n+1}$ так, чтобы центр шара $X^{k}$ совпадал с началом координат и касательная плоскость к поверхности $S$ в точке $X^{k}:=\left(\left(x^{k}, f\left(x^{k}\right)\right)\right.$ определялась уравнением $x_{n+1}=0$. Иньми словами, $S$ задается в виде графика функции $x_{n+1}=f(x)$ со свойством $\nabla f(0)=0$. Более того, функция $f$ посредством врашения записьвается в виде

$$
f(x)=\sum_{j=1}^{n} \lambda_{j} x_{j}^{2}+\sum_{|\alpha|=3} x^{\alpha} H_{\alpha}(x),
$$

где $H_{\alpha}$ - гладкие функции.

Заметим, что $\Lambda_{1}(X)$ и $\Lambda_{2}(X)$ - инвариантные функции относительно евклидова движения. В частности, мы можем вычислить $\Lambda_{1}(X)$ и $\Lambda_{2}(X)$ в новой системе координат. В результате для них получим следующие соотношения:

$$
\Lambda_{1}\left(\lambda_{1} x\right)=\lambda_{1}^{2} h_{1}(x, \lambda), \quad \Lambda_{2}\left(\lambda_{1} x\right)=\lambda_{1}^{4} h_{2}(x, \lambda),
$$

где $h_{1}, h_{2}$ - вещественно-аналитические функции от $(x, \lambda)$.

В интеграле $I_{k}(t, s)$ сделаем замену переменных $x \mapsto \lambda_{1} x$ и с учетом вьшеприведенных тождеств имеем

$$
I_{k}(t, s)=\left|\lambda_{1}\right|^{n+q} \int_{\{|x|<c\}} e^{i t \lambda_{1}^{3} F_{1}(x, \sigma)}(h(x, \sigma))^{q} \Phi_{k}(x, \lambda) d x,
$$

где

$$
\begin{gathered}
F_{1}(x, \sigma)=\sum_{j=1}^{n} \frac{\lambda_{j}}{\lambda_{1}} x_{j}^{2}+\sum_{|\alpha|=3} x^{\alpha} H_{\alpha}(x, \lambda)+(\sigma, x), \quad \sigma=\frac{s}{\lambda_{1}^{2}}, \\
\Phi_{k}(x, \lambda)=\psi(x, \lambda) \psi_{k}(x, \lambda), \quad h(x, \lambda)=h_{1}(x, \lambda)^{q_{1} / 2} h_{2}(x, \lambda)^{q_{2} / 4} .
\end{gathered}
$$

Заметим, что функция

$$
f_{1}(x, \lambda)=\sum_{j=1}^{n} \frac{\lambda_{j}}{\lambda_{1}} x_{j}^{2}+\sum_{|\alpha|=3} x^{\alpha} H_{\alpha}(x, \lambda)
$$

удовлетворяет условиям леммы 4.3 , причем $\left\|H_{\alpha}\right\|_{k}$ оценивается через $\|f\|_{k+3}$. 
Пусть $\varepsilon$ - достаточно малое положительное число. Тогда уравнение $\partial_{1} F_{1}(x, \sigma)=0$ имеет единственное аналитическое решение $x_{1}\left(x^{\prime}, \sigma_{1}\right)$. Через $I^{\prime}$ обозначается идеал, порожденный элементами матрицы $D_{x^{\prime}}^{2} F\left(x_{1}\left(x^{\prime}, \sigma_{1}\right), x^{\prime}, \sigma\right)$. Легко видеть, что функция $h_{2}\left(x_{1}\left(x^{\prime}, \sigma_{1}\right), x^{\prime}, \sigma\right)$ принадлежит идеалу $I^{\prime}$.

Теперь для параметра $\sigma_{1}$ рассмотрим два случая.

Случай 1: $\left|\sigma_{1}\right| \geqslant \varepsilon$, где числа $\varepsilon$ и $c$ выбираются согласно лемме 4.3. В этом случае, используя лемму Ван дер Корпута [21], [22], получим

$$
\left|I_{k}(t, s)\right| \leqslant \frac{C\left|\lambda_{1}\right|^{n+q}}{\left(1+\left|t \lambda_{1}^{3}\right|\right)} .
$$

Следовательно, для $\delta=\min \{1 / 2,(2 q-3) / 6\}$ имеем оценку

$$
\left|I_{k}(t, s)\right| \leqslant \frac{C\left|\lambda_{1}\right|^{n}}{|t|^{1 / 2+\delta}} .
$$

Случай $2:\left|\sigma_{1}\right|<\varepsilon$. Тогда, используя лемму 4.3, интеграл $I_{k}(t, s)$ приводим к виду

$$
\begin{aligned}
I_{k}(t, s)= & \left|\lambda_{1}\right|^{n+q} \int_{\mathbb{R}^{n-1}} e^{i t \lambda_{1}^{3} F\left(\left(x_{1}\left(x^{\prime}, \sigma_{1}\right), x^{\prime}, \sigma\right)\right.} d x^{\prime} \\
& \times \int_{\mathbb{R}} e^{i t \lambda_{1}^{3}\left(x_{1}-x_{1}\left(x^{\prime}, \sigma_{1}\right)\right)^{2} \Phi\left(x, \sigma_{1}\right)}(h(x, \lambda))^{q} a(x, \lambda, \sigma) d x_{1} .
\end{aligned}
$$

Теперь мы используем следующее элементарное соотношение:

$$
\begin{aligned}
h_{1}\left(x, \lambda_{1}\right)^{q_{1}^{\prime}} h_{2}\left(x, \lambda_{1}\right)^{q_{2}^{\prime}=} & \left(h_{1}\left(x, \lambda_{1}\right)^{q_{1}^{\prime}}-h_{1}\left(x_{1}\left(x^{\prime}, \sigma_{1}\right), x^{\prime}, \lambda_{1}\right)^{q_{1}^{\prime}}\right) h_{2}\left(x, \lambda_{1}\right)^{q_{2}^{\prime}} \\
& +h_{1}\left(x_{1}\left(x^{\prime}, \sigma_{1}\right), x^{\prime}, \lambda_{1}\right)^{q_{1}^{\prime}}\left(h_{2}\left(x, \lambda_{1}\right)^{q_{2}^{\prime}}-h_{2}\left(x_{1}\left(x^{\prime}, \sigma_{1}\right), x^{\prime}, \lambda_{1}\right)^{q_{2}^{\prime}}\right) \\
& +h_{1}\left(x_{1}\left(x^{\prime}, \sigma_{1}\right), x^{\prime}, \lambda_{1}\right)^{q_{1}^{\prime}} h_{2}\left(x_{1}\left(x^{\prime}, \sigma_{1}\right), x^{\prime}, \lambda_{1}\right)^{q_{2}^{\prime}},
\end{aligned}
$$

где $q_{1}^{\prime}=q q_{1} / 2$ и $q_{2}^{\prime}=q q_{2} / 4$. Применяя лемму 3.4 к следующей разности, получим

$$
\left(h\left(x, \lambda_{1}\right)\right)^{q}-\left(h\left(x_{1}\left(x^{\prime}, \sigma_{1}\right), x^{\prime}, \lambda_{1}\right)\right)^{q}=\left|x_{1}-x_{1}\left(x^{\prime}, \sigma_{1}\right)\right|^{q_{0}} b\left(x, \sigma_{1}\right),
$$

где $q_{0}=\min \left\{q_{1}^{\prime}, q_{2}^{\prime}\right\}$. Заметим, что $b\left(x, \sigma_{1}\right)$ имеет ограниченную вариацию и ее полная вариация по $x_{1}$ равномерно ограничена относительно остальных переменных. Следовательно, мы можем представить наш интеграл в виде суммы двух интегралов

$$
I_{k}(t, s)=I_{1 k}(t, s)+I_{2 k}(t, s)=I_{1 k}+I_{2 k},
$$

где

$$
\begin{gathered}
I_{2 k}=\left|\lambda_{1}\right|^{n+q} \int_{\mathbb{R}^{n-1}} e^{i t \lambda_{1}^{3} F\left(\left(x_{1}\left(x^{\prime}, \sigma_{1}\right), x^{\prime}, \sigma\right)\right.} d x^{\prime} \\
\times \int_{\mathbb{R}} e^{i t \lambda_{1}^{3}\left(x_{1}-x_{1}\left(x^{\prime}, \sigma_{1}\right)\right)^{2} \Phi\left(x, \sigma_{1}\right)}\left|x_{1}-x_{1}\left(x^{\prime}, \sigma_{1}\right)\right|^{q_{0}} a_{1} d x_{1}, \\
a_{1}:=a_{1}\left(x, \lambda, \sigma_{1}\right)=b\left(x, \sigma_{1}\right) a(x, \lambda), \\
I_{1 k}=\left|\lambda_{1}\right|^{n+q} \int_{\mathbb{R}^{n-1}} e^{i t \lambda_{1}^{3} F\left(\left(x_{1}\left(x^{\prime}, \sigma_{1}\right), x^{\prime}, \sigma\right)\right.}\left(h\left(x_{1}\left(x^{\prime}, \sigma_{1}\right), x^{\prime}, \lambda_{1}\right)\right)^{q} d x^{\prime} \\
\times \int_{\mathbb{R}} e^{i t \lambda_{1}^{3}\left(x_{1}-x_{1}\left(x^{\prime}, \sigma_{1}\right)\right)^{2} \Phi\left(x, \sigma_{1}\right)} a(x, \lambda, \sigma) d x_{1} .
\end{gathered}
$$

Мы можем использовать оценку типа Эрдейи [4]. 
ЛЕмма 5.1. Если функиия а имеет ограниченную вариацию на $\mathbb{R} u 0<q<1$ фиксированное вещественное число, то существует постоянная $C$ такая, что справедлива следующая оценка:

$$
\left.\left|\int_{\mathbb{R}}\right| x\right|^{q} a(x) e^{i t x^{2}} d x \mid \leqslant \frac{C\|a\|_{V}}{|t|^{(1+q) / 2}},
$$

где $\|a\|_{V}=|a(-\infty)|+V_{\mathbb{R}}[a]-$ вариационная норма.

Согласно лемме 5.1 получим

$$
\left|I_{1 k}(t, s)\right| \leqslant c \frac{\left|\lambda_{1}\right|^{n}\|a\|_{L_{3}^{1}}}{|t|^{1 / 2+\delta}} .
$$

Далее, мы рассмотрим внутренний интеграл

$$
J_{k}:=\int_{\mathbb{R}} e^{i t \lambda_{1}^{3}\left(x_{1}-x_{1}\left(x^{\prime}, \sigma_{1}\right)\right)^{2} \Phi\left(x, \sigma_{1}\right)} a(x, \lambda, \sigma) d x_{1} .
$$

Заметим, что амплитуда последнего интеграла - гладкая функция. Следовательно, мы можем использовать обычный метод стационарной фазы и получим [23]:

$$
J_{k}=c\left|t \lambda_{1}^{3}\right|^{-1 / 2} a\left(x_{1}\left(x^{\prime}, \sigma_{1}\right), x^{\prime}, \sigma_{1}\right)+R\left(t, \lambda_{1}, x^{\prime}, \sigma_{1}\right) .
$$

Для остаточного члена мы имеем следующую равномерную оценку [24]:

$$
\left|R\left(t, \lambda_{1}, x^{\prime}, \sigma_{1}\right)\right| \leqslant c \frac{\left|\lambda_{1}\right|^{n}\|a\|_{L_{3}^{1}}}{\left|t \lambda_{1}^{3}\right|^{3 / 2}} .
$$

Последнее неравенство дает искомую оценку для остаточного члена.

Наконец, рассмотрим вклад главного члена асимптотического разложения. Для этого преждемы рассмотрим демпфированньй интеграл в более общей форме. Пусть $f(x, \eta)$ - вещественно-аналитическая функция в начале координат $\mathbb{R}^{n} \times \mathbb{R}^{m}$. Введем идеал $I$ кольца ростков аналитических функций в начале координат, порожденный элементами матрицы $D_{x}^{2} f(x, \eta)$.

Через $F(x, \eta, s)$ обозначим фазовую функцию, определенную по формуле

$$
F(x, \eta, s)=f(x, \eta)+s x .
$$

Теперь рассмотрим следующий демпфированньй осцилляторньй интеграл:

$$
T(t, \eta, s)=\int_{\mathbb{R}^{n}} e^{i t F(x, \eta, s)} a(x, \eta, s)|\varphi(x, \eta)|^{q} d x,
$$

где $\varphi \in I$.

ПРЕДЛОЖЕНИЕ 5.2. Пусть $\varphi \in I$ - фиксированный әлемент идеала и $q$ - фиксированное положительное число. Существуют окрестность нуля $U \times V \times W \subset$ $\mathbb{R}^{n} \times \mathbb{R}^{m} \times \mathbb{R}^{n}$ и положительное число $\varepsilon>0$ такие, что для любой амплитудной функиии $а \in C_{0}^{\infty}(U \times V \times W)$ справедлива следующая оченка:

$$
|T(t, \eta, s)| \leqslant c \frac{\|a(\cdot, \eta, s)\|_{L_{3}^{1}}}{|t|^{\varepsilon}} .
$$


ЗАмЕчАниЕ 5.1. Следует отметить, что если $d f(x, 0) \not \equiv 0$, то согласно обобщенной лемме Ван дер Корпута для кратных интегралов искомое неравенство вьполняется без всякого множителя гашения $|\varphi|^{q}[21],[22]$.

Как показьвают примеры в случае $d f(x, 0) \equiv 0$, аналогичная оценка без множителя гашения не верна.

ДОКАЗАТЕЛЬСТВО ПРЕДЛОЖЕНИЯ 5.2. Пусть $f(0,0)=0$ и $D_{x}^{2} f(0,0)=0$. Если $K(I)=U \times V$ (или $I=0)$, то доказывать нечего, так как в этом случае $\varphi=0$. Далее, предположим, что $K(I)$ - некоторое собственное аналитическое множество. Пусть $\eta \in V$ - фиксированная точка. Рассмотрим множество $U_{0}(\eta)=\left\{x: D_{x}^{2} f(x, \eta) \neq 0\right\}$. Если это множество пусто, то $T(t, \eta, s)=0$ для любого $(t, s)$. Предположим, что $U_{0}(\eta) \neq \varnothing$. В этом случае мы можем использовать лемму 4.2 и получить покрытиемножества $U_{0}(\eta)$ с помощью шаров $\left\{B_{k}\right\}$ (фактически $B_{k}:=B_{k}^{\prime}$ в этой лемме). Пусть $\left\{\psi_{k}\right\}-$ разбиение единищы, соответствующее этому покрытию. С помощью этого разбиения единицы наш интеграл $T(t, \eta, s)$ представляется в виде суммы интегралов:

$$
T(t, \eta, s)=\sum_{l} T_{l}(t, \eta, s)
$$

Рассмотрим оценку одного из членов суммы

$$
T_{l}(t, \eta, s)=\int_{\mathbb{R}^{n}} e^{i t F(x, \eta, s)} a(x, \eta, s) \psi_{l}(x)|\varphi(x, \eta)|^{q} d x .
$$

Заметим, что $\max _{\nu}\left|\lambda_{\nu}(x, \eta)\right| \asymp\left\|D_{x}^{2} f(x, \eta)\right\|$, где $\left\{\lambda_{\nu}(x, \eta)\right\}_{\nu=1}^{n}$ рассматриваются как главные кривизны гиперповерхности, определяемой графиком функции $f(\cdot, \eta)$. Используем замену переменных $x(y)=x^{l}+\left\|D_{x}^{2} f(x, \eta)\right\| y$ и получим

$$
\begin{aligned}
T_{l}(t, \eta, s)= & \left\|D_{x}^{2} f(x, \eta)\right\|^{n} e^{i t F\left(x^{l}, \eta, s\right)} \\
& \times \int_{\mathbb{R}^{n}} e^{i t F_{1}(x(y), \eta, s)} a(x(y), \eta, s) \psi_{l}(x(y))|\varphi(x(y), \eta)|^{q} d y .
\end{aligned}
$$

Мы можем представить нашу фазовую функцию в следующем виде:

$$
F_{1}(x(y), \eta, s)=\left\|D_{x}^{2} f(x, \eta)\right\|^{3}\left(\left(\frac{D_{x}^{2} f(x, \eta) y}{\left\|D_{x}^{2} f(x, \eta)\right\|}, y\right)+O\left(|y|^{3}\right)+(\sigma, y)\right),
$$

где $\sigma=D_{x}^{2} f(x, \eta) y /\left\|D_{x}^{2} f(x, \eta)\right\|^{2}$. Более того, асимптотика $O\left(|y|^{3}\right)$ равномерна относительно параметра $\eta$. Введем новую фазовую функцию

$$
f_{2}(y, \eta)=\frac{F_{1}(x(y), \eta, 0)}{\left\|D_{x}^{2} f(x, \eta)\right\|^{3}}
$$

Очевидно, справедливо следующее неравенство:

$$
\left\|D_{y}^{2} f_{2}(y, \eta)\right\| \geqslant 1
$$

для любого $y \in\{|y|<c\}$. Согласно лемме 4.4 максимальное значение и полная вариация функции $|\varphi(x(y), \eta)|^{q}$ по множеству $\left\{\left|y_{l}\right| \leqslant c\right\}$ оценивается через $C\left\|D_{x}^{2} f(x, \eta)\right\|^{q}$. 
Следовательно, из оценки типа Ван дер Корпута [21], [22], [25] в случае $k=2$ при $\delta \leqslant 1 / 2$ имеем

$$
\left|T_{l}(t, \eta, s)\right| \leqslant \frac{C\left\|D_{x}^{2} f\left(x^{l}, \eta\right)\right\|^{n+q}\|a\|_{L_{3}^{1}}}{|t|\left|D_{x}^{2} f\left(x^{l}, \eta\right) \|\right|^{\delta}} .
$$

Из последнего неравенства вытекает оценка

$$
\left|T_{l}(t, \eta, s)\right| \leqslant \frac{C\left\|D_{x}^{2} f\left(x^{l}, \eta\right)\right\|^{n}\|a\|_{L_{3}^{1}}}{|t|^{\varepsilon}}
$$

где $\varepsilon=\min \{1 / 2, q / 2\}$.

Заметим, что ряд

$$
\sum_{l}\left\|D_{x}^{2} f\left(x^{l}, \eta\right)\right\|^{n}
$$

сходится согласно лемме 4.2 .

Итак, суммируя полученные оценки по всем индексам $l$, мы получим доказательство предложения 5.2.

Теперь рассмотрим оценку для интеграла $\tilde{I}_{k}(t, s)$, где $\tilde{I}_{k}(t, s)$ - вклад главной части асимптотики интеграла $I_{1 k}(t, s)$ :

$$
\begin{gathered}
\tilde{I}_{k}(t, s)=c \frac{\left|\lambda_{1}\right|^{n+q}}{\left|t \lambda_{1}^{3}\right|^{1 / 2}} \int_{\mathbb{R}^{n-1}} e^{i t F_{1}\left(x^{\prime}, \eta, \sigma\right)}\left(h_{1}\left(x_{1}\left(x^{\prime}, \sigma_{1}\right), x^{\prime}, \lambda_{1}\right)\right)^{q_{1}^{\prime}} \\
\times\left(h_{2}\left(x_{1}\left(x^{\prime}, \sigma_{1}\right), x^{\prime}, \lambda_{1}\right)\right)^{q_{2}^{\prime}} a_{2} d x^{\prime},
\end{gathered}
$$

где

$$
a_{2}:=a_{2}\left(x^{\prime}, \lambda, \sigma_{1}\right)=a_{1}\left(x_{1}\left(x^{\prime}, \sigma_{1}\right), x^{\prime}, \lambda, \sigma_{1}\right) .
$$

Как было отмечено вьше, $h_{2}\left(x_{1}\left(x^{\prime}, \sigma_{1}\right), x^{\prime}, \lambda_{1}\right) \in I$, где $I$ - идеал, порожденньй элементами матрищы $D_{x^{\prime}}^{2} F_{1}\left(x^{\prime}, \eta, \sigma\right)$, и $q_{2}^{\prime}>0$. Здесь мы существенно используем тот факт, что $q_{2}>0$. Теперь мы используем предложение 5.2. В силу этого предложения для достаточно малого положительного числа $\delta>0$ выполняется оценка

$$
\left|\tilde{I}_{k}(t, s)\right| \leqslant \frac{C\left|\lambda_{1}\left(x^{k}\right)\right|^{n+q-3 / 2-\delta}\|a\|_{L_{3}^{1}}}{|t|^{1 / 2+\delta}}
$$

Заметим, что мы можем использовать лемму Ван дер Корпута, так как согласно лемме 3.2 функция $\left(h_{1}\left(x_{1}\left(x^{\prime}, \sigma_{1}\right), x^{\prime}, \lambda_{1}\right)\right)^{q_{1}^{\prime}}$ имеет ограниченную вариацию относительно $x_{k}, k=2, \ldots, n$. Теперь берем $\varepsilon=q-(3 / 2+\delta)$, предполагая вьполнение неравенства $0<\delta<q-3 / 2$. Суммируя полученные неравенства по всем индексам $k$ и учитывая сходимость ряда $\sum_{k}\left|\lambda_{1}\left(x^{k}\right)\right|^{n}$, получим оценку

$$
|I(t, s)| \leqslant \frac{C\|a\|_{L_{3}^{1}}}{|t|^{1 / 2+\varepsilon}}
$$

Последняя оценка доказьвает теорему 2.1 .

В заключение автор выражает свою глубокую благодарность профессору В. П. Паламодову за ценные советы и полезные обсуждения полученных результатов, а также рецензенту за ценные замечания.

Работа была завершена во время визита автора в Международный центр теоретической физики им. Абдус Салама (г. Триест, Италия). Автор благодарит профессора А. О. Куку за полезные обсуждения результатов. 


\section{СПИСОК ЦИТИРОВАННОЙ ЛИТЕРАТУРЫ}

[1] Stein E. M. Maximal functions: spherical means // Proc. Nat. Acad. Sci. USA. 1976. V. 73. P. 2174-2175.

[2] Bourgain J. Averages in the plane convex curves and maximal operators // J. Anal. Math. 1986. V. 47. P. 69-85.

[3] Sogge C. D., Stein E. M. Averages of functions over hypersurfaces in $\mathbb{R}^{n} / /$ Invent. Math. 1985. V. 82. P. 543-556.

[4] Stein E. M. Harmonic Analysis: Real-Valued Methods, Orthogonality, and Oscillatory Integrals. Princeton: Princeton Univ. Press, 1993.

[5] Greenleaf A. Principal curvature and harmonic analysis // Indiana Math. J. 1982. V. 30. P. 519-537.

[6] Sogge C. D. Averaging operators with one non-vanishing principal curvature // Fourier Analysis and Partial Differential Equations. Stud. Adv. Math. 1995. V. 22. P. 317-323.

[7] Cowling M., Mauceri G. Inequalities for maximal functions II // Trans. Amer. Math. Soc. 1986. V. 296. P. 341-365.

[8] Cowling M., Mauceri G. Oscillatory integrals and Fourier transforms of surface carried measures // Trans. Amer. Math. Soc. 1987. V. 304. № 1. P. 53-68.

[9] Iosevich A. Maximal operators associated to families of flat curves in the plane // Duke Math. J. 1995. V. 76. P. 631-644.

[10] Iosevich A., Sawyer E. Maximal operators over convex hypersurfaces // Adv. in Math. 1997. V. 132. № 1. P. 46-119.

[11] Арнольд В.И., Варченко А.Н., Гусейн-заде С. М. Особенности дифференцируемых отображений. Ч. 1. Классификация критических точек каустик и волновых фронтов. М.: Наука, 1982.

[12] Варченко А.Н. Многогранники Ньютона и оценки осциллирующих интегралов // Функцион. анализ и его прилож. 1976. Т. 10. № 3. С. 13-38.

[13] Карпушкин В.Н.// Теорема о равномерной оценке осциллирующих интегралов с фазой, зависящей от двух переменных. Труды семин. им. И. Г. Петровского. 1984. Т. 10. С. 150-169.

[14] Cowling M., Disney S., Mauceri G., Müller D. Damping oscillatory integrals // Invent. Math. 1990. V. 101. P. 237-260.

[15] Ikromov I. A. Damped oscillatory integrals and boundedness problem for maximal operators // Preprint IC/2003/43. Italy: ICTP Trieste, 2003.

[16] Дубровин Б. А., Новиков С. П., Фоменко А. Т. Современная геометрия. М.: Наука, 1986.

[17] Hartman P., Nirenberg L. On spherical image maps whose Jacobians do not change sign // Amer. J. Math. 1959. V. 81. P. 901-920.

[18] Malgrange B. Ideals of Differentiale Functions. Oxford: Oxford Univ. Press, 1966.

[19] Hironaka H. Resolution of singularities of an algebraic variety over a field of characteristic zero. I, II // Ann. of Math. 1964. V. 79. № 2. P. 109-326.

[20] Duistermaat J. Oscillatory integrals, Lagrange immersions and unfolding of singularities // Comm. Pure Appl. Math. 1974. V. 27. № 2. P. 207-281.

[21] Архипов Г. И., Карацуба А. А., Чубариков В. Н. Тригонометрические интегралы // Изв. АН СССР. Сер. матем. 1979. Т. 43. № 5. С. 971-1003.

[22] Carbery A., Christ M., Wright J. Multidimensional van der Corput lemma and sublevel set estimates // J. Amer. Math. Soc. 1999. V. 12. № 4. P. 981-1015.

[23] Федорюк М. В. Метод перевала. М.: Наука, 1977.

[24] Попов Д. А. Оценки с константами для некоторых классов осциллирующих интегралов // УМH. 1997. Т. 52. №1. С. 77-148.

[25] Van der Corput J. G. Zahlentheoretische Abschätzungen // Math. Ann. 1921. V. 84. P. 53-79. 